\title{
Data Change Date
}

National Cancer Institute

\section{Source}

National Cancer Institute. Data Change Date. NCI Thesaurus. Code C114550.

The date of data modification. 